\title{
BIOQUÍMICA DE ALIMENTOS NA WEB: PROPOSTA DE UM SITE DE APOIO ÀS AULAS EXPERIMENTAIS PRESENCIAIS OU A DISTÂNCIA
}

\author{
Karina Aparecida de Freitas Dias de Souza ${ }^{1}$; Valdir Augusto Neves ${ }^{2}$ \\ ${ }^{1}$ Departamento de Química Analítica do Instituto de Química da UNESP, Araraquara; \\ ${ }^{2}$ Departamento de Alimentos e Nutrição da Faculdade de Ciências Farmacêuticas da \\ UNESP, Araraquara. \\ *kfreitas@iq.unesp.br
}

\begin{abstract}
RESUMO
Com base na evidente falta de recursos para a realização de atividades experimentais especialmente em estabelecimentos públicos de ensino médio regular e técnico, e no crescimento acentuado no uso de computadores na escola, o presente trabalho apresenta um guia de aulas práticas de Bioquímica, que tem por objetivo complementar as aulas presenciais, não substituindo outras matérias nem servindo como único contato dos estudantes com as práticas laboratoriais, mas contribuindo com uma ferramenta de aprendizado no auxílio ao discente e ao docente. O site apresenta-se sob a forma de textos interativos, incluindo uma introdução teórica aos tópicos de interesse à Bioquímica de Alimentos e roteiros para a realização dos experimentos, sempre acompanhados de uma seqüência de fotografias demonstrativas que permitem ao usuário acompanhar o experimento virtualmente. $\mathrm{O}$ site encontra-se hospedado no portal da UNESP, no endereço http://www.fcfar.unesp.br/alimentos/bioquimica/menu.htm, onde foram disponibilizados formulários, que objetivam a avaliação e constante melhora do material.
\end{abstract}

\begin{abstract}
Based on the evident lack of resources to the realization of experimental activities, especially in the Brazilian public high school establishments, and observing a considerable increase in the scholar use of computers, this paper presents an electronic guide containing biochemical experiments, that intends to complement the actual classes, neither substituting other kinds of subjects, nor serving as the students unique contact with the practical activities, but contributing as a teaching and learning tool. The site brings up interactive texts, including a theoretical introduction to important topics in food biochemistry and the description of the experimental proceedings, which always shows a demonstrative photographic sequence that allows the virtual accompaniment of the experiments. This website can be accessed by the virtual address http://www.fcfar.unesp.br/alimentos/bioquimica/menu.htm, where forms are available, aimed for evaluation and constant material improvement.
\end{abstract}




\section{INTRODUÇÃO}

\section{A experimentação na escola}

A introdução do trabalho experimental nas escolas teve seu início há mais de cem anos, sob influência das atividades científicas desenvolvidas nas universidades [1]. $O$ objetivo dessa medida era a aplicação do conhecimento teórico adquirido, para melhorar a aprendizagem do conteúdo científico. Hoje, o papel fundamental da experimentação no ensino de ciências é praticamente consenso entre os professores.

O desenvolvimento de habilidades e competências, a melhor compreensão da teoria discutida e a possibilidade de encontrar e analisar fatos por meio da investigação foram alguns dos motivos apontados para a inclusão de experimentos nas aulas de ciências em trabalho publicado por Kerr em 1963 [1], período pós Segunda Grande Guerra, época de maior incentivo à realização de atividades práticas na escola. Esses mesmos apontamentos continuam embasando as argumentações em favor dessa prática metodológica em trabalhos publicados mais recentemente [2].

Apesar de algumas pesquisas criticarem duramente alguns dos motivos expostos acima [2-3], fica claro que a experimentação permanece viva no ambiente escolar, onde apresenta caráter motivador e lúdico, estando essencialmente atrelada aos sentidos [4].

\section{As atividades escolares de experimentação no Brasil}

Apesar da reconhecida importância do trabalho prático na educação básica, o Brasil parece pouco investir na melhoria e construção de espaços físicos destinados ao desenvolvimento dessas atividades. A pesquisa EDUDATABRASIL (2005) [5] revelou que apenas $38 \%$ das escolas públicas que oferecem o Ensino Médio (ambiente urbano e rural) possuem laboratório de ciências, valor contrastado com o índice de 65,6\% para as escolas particulares; em 1999 esses números correspondiam a 39 e 61\%, respectivamente. Isso evidencia a ausência de ambiente físico adequado para a realização de experimentos, principalmente no que se refere aos estabelecimentos públicos de ensino, além de sua desprezível evolução no período. Esse fato, por si só, não afetaria o desenvolvimento de atividades práticas: apesar de não terem sido encontrados dados sobre isso, é possível constatar que a falta de recursos não se restringe ao espaço físico, mas também à aquisição de materiais, o que limita ainda mais a ação dos professores.

Em contrapartida, se, para o mesmo período, for avaliado o investimento na informatização da escola (presença de microcomputadores e laboratórios de informática, além do acesso à internet), a realidade encontrada será um tanto diferente. A Figura 1 mostra uma crescente presença da tecnologia nas escolas, cada vez mais incentivada pelos órgãos governamentais e demais instituições responsáveis pelo ensino médio no Brasil. Os laboratórios de ciências, em contrapartida, registraram uma redução no mesmo período.

Diante desse quadro e da relevância da experimentação na construção de conceitos científicos na educação básica, é de fundamental importância a criação de alternativas à existência de laboratórios de ensino. Nesse contexto, a tecnologia pode contribuir muito, suprindo, em parte, a falta do espaço físico e equipamentos mais adequados. 


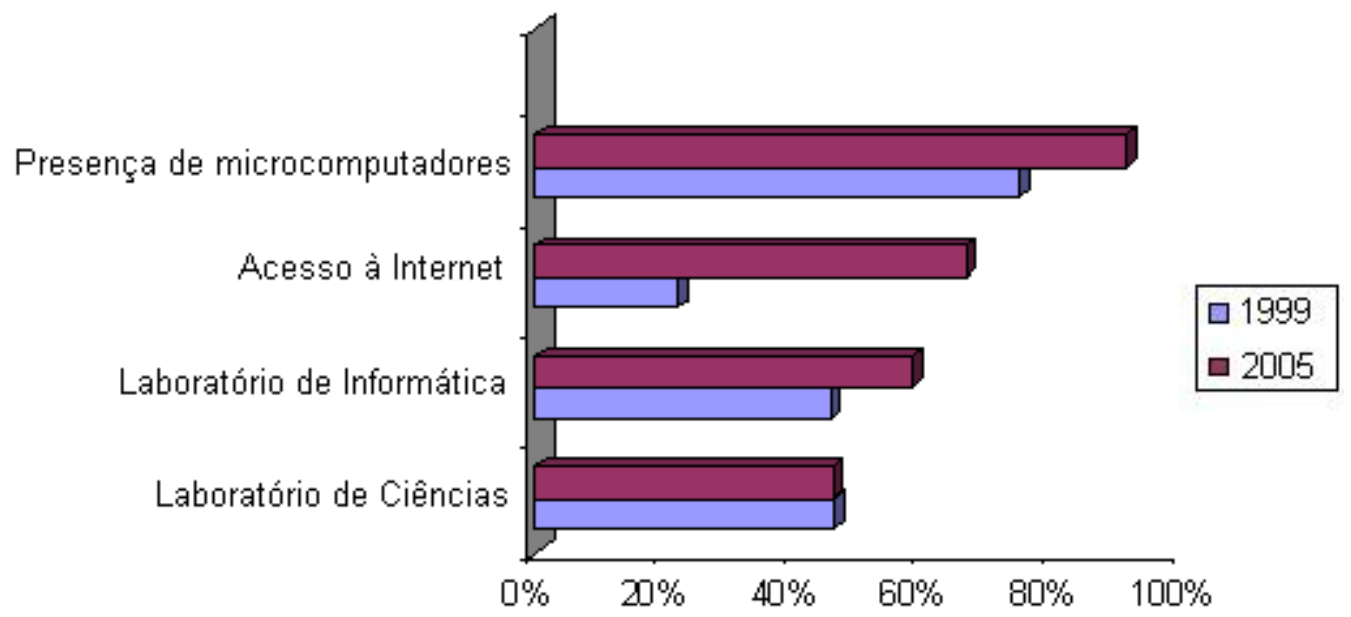

Figura1. A presença de laboratórios de ciências frente ao investimento na informatização em estabelecimentos (públicos e particulares) que oferecem o Ensino Médio (EDUDATABRASIL, 2005).

\section{Tecnologia e educação}

Apesar da expressão "tecnologia educacional" remeter imediatamente à utilização de instrumentos e equipamentos modernos no processo educativo, a tecnologia e a sala de aula caminham lado a lado há muito tempo. Segundo Saettler [6], o uso da tecnologia na educação já se caracterizava nas pictografias e sinais de escrita usados para gravar ou transmitir informações. Assim, a tecnologia educacional é, essencialmente, o produto de um longo percurso histórico, marcado por inúmeros ciclos de tentativa e erro. Essa característica processual faz com que pesquisadores da área rejeitem o conceito reducionista de que a tecnologia educacional é a utilização de instrumentos como auxiliares no ensino. Mais que isso: é necessário priorizar o processo de aplicação dessas ferramentas dentro das propostas educacionais [7].

Ao longo das décadas, foram várias as tecnologias aplicadas nas salas de aula, dentre as quais estão o caderno, o lápis, o giz, a lousa e os livros impressos. Todas elas tiveram sua gradual participação na mudança de hábitos e comportamentos de estudantes e professores. Porém, o quadro vivenciado pelo mundo nos últimos anos é um pouco diferente: o de acelerada revolução nas tecnologias e sistemas de comunicação, que se desenvolvem e diversificam sem parar, impedindo qualquer previsão das mudanças que serão causadas na sociedade [8].

As conseqüências (positivas e negativas) desses avanços já vêm se fazendo sentir na área educacional. Diante do intenso processo evolutivo na área tecnológica, foi necessária a criação de novos termos que melhor se adequassem aos acontecimentos. O termo novas tecnologias da informação (TIC), proposto pelo Ministério da Educação [9], agrega tecnologias que foram produzidas no contexto de outras relações sociais, para outros fins [10], mas que são importantes recursos auxiliares no processo de ensino-aprendizagem, uma vez que, de uma forma ou de outra, já estão presentes no dia-a dia dos alunos. Fazem parte desse grupo a televisão, o rádio, o vídeo, o computador, a Internet, etc. [11]. Os próprios Parâmetros Curriculares Nacionais [12] enfatizam que as tecnologias educacionais, em especial o computador, permitem novas formas de trabalho, possibilitando a criação de ambientes de aprendizagem em que os alunos pesquisam, fazem antecipações e simulações, confirmam idéias prévias, experimentam, criam soluções e constroem novas formas de representação mental. 


\section{A utilização do computador no processo de ensino-aprendizagem}

O ensino através da informática tem suas raízes no ensino através das máquinas proposto por Pressey em 1924, e no conceito de instrução programada proposto por Skinner em 1950, dentro do qual se dividia o material a ser ensinado em módulos, sendo apresentada uma questão ao final de cada um deles [13].

Salvo as críticas aplicadas a esse modelo [14], a instrução programada era apresentada na forma impressa e foi muito utilizada no final da década de 50 e início dos anos 60 [13]. Nas escolas, a disseminação desses programas só foi possível com os microcomputadores, ainda na década de 60, que permitiram uma enorme produção de cursos e uma diversificação dos tipos de programas utilizados. Em 1983 foram identificados mais de 7000 pacotes de softwares educacionais no mercado, sendo que 125 eram adicionados a cada mês [13].

Apesar da reconhecida importância da utilização dos recursos de informática desde a década de 60, os professores brasileiros só tiveram os computadores como aliados nas salas de aula no começo dos anos 80 [15], processo que se iniciou nas escolas particulares, estendendo-se mais tarde para as escolas públicas.

A tecnologia não parou de avançar e, no panorama tecnológico atual, existem diversas alternativas para a aprendizagem com o uso do computador. Novas designações foram criadas para abranger os novos avanços, como por exemplo, o termo tecnologias interativas aplicadas ao ensino, que se refere fundamentalmente aos meios instrucionais baseados nos mais recentes avanços computacionais como programas interativos, CD-ROM, hipertexto, hipermídia, correio eletrônico (e-mail), realidade virtual, simuladores, cursos à distância, entre outros [8].

No que se refere ao ensino de Bioquímica, tema de especial interesse para esse trabalho, diversos materiais que se utilizam das tecnologias interativas de ensino encontram-se disponíveis na literatura. Dentre eles, são especialmente interessantes os softwares que se dedicam à modelagem de fenômenos e sistemas $[16,17]^{1}$, dado que favorecem a construção do conhecimento no nível teórico-conceitual, propiciando a construção de modelos por parte dos estudantes [18]. Além disso, a disponibilização eletrônica de conteúdos e a utilização dos recursos da informática enquanto forma de complementação desses conteúdos, o que pode ser feito adotando-se estratégias mais simples, como a construção de sites, exerce importante papel na disponibilização irrestrita do conteúdo, facilitando o acesso indistinto ao conhecimento. No tocante às atividades experimentais, essas, quando presentes, vêm sendo apresentadas nos materiais disponíveis na literatura essencialmente na forma de roteiros descritivos que em muitas das vezes requerem materiais de difícil acesso, especialmente, para as escolas públicas onde os recursos apresentam-se escassos.

Preservando a compreensão de que a tecnologia não funciona automaticamente como desencadeadora do processo de aprendizagem, mas que pode criar condições que facilitem esse processo [13], e guiado pela preocupante falta de recursos para realização de experimentos nos níveis médio e profissionalizante de ensino, o presente trabalho é resultado da construção de um guia de aulas práticas em Bioquímica de Alimentos, destinado, inicialmente, a professores e alunos de cursos da área de Alimentos e Nutrição, o qual permite a visualização de fenômenos que dependeriam de condições físicas e materiais adequados para tal. $O$ material encontra-se disponibilizado na forma de um site, e dispõe de textos introdutórios,

\footnotetext{
${ }^{1}$ Outras referências e links relativos à produção de materiais destinados ao ensino de Bioquímica encontram-se disponibilizados no site da Revista Brasileira de Ensino de Bioquímica e Biologia Molecular (http://www.sbbq.org.br/revista/)
} 
roteiros e atividades práticas fotografadas, conforme será apresentado posteriormente. É importante destacar que o material aqui apresentado não apresenta pretensões pedagógicas, sendo essencial a participação do professor enquanto mediador do processo educativo, e servindo apenas como alternativa à escassez de recursos, no sentido de contribuir para o preenchimento das lacunas abertas por ela.

\section{Desenvolvimento do trabalho}

Seguindo os moldes de um programa tutorial, o site apresenta-se na forma de textos interativos, incluindo uma introdução teórica à Bioquímica de Alimentos e roteiros para a realização dos experimentos, sempre acompanhados de uma seqüência de fotografias demonstrativas, visando alcançar aqueles que não possuem a disponibilidade de execução do mesmo. Sendo assim, o trabalho foi desenvolvido em três etapas principais, a saber:

Construção dos textos de apoio teórico;

Pesquisa das atividades práticas de interesse na área de Bioquímica de Alimentos passíveis de serem desenvolvidas e elaboração dos roteiros, guia para a realização dessas atividades; e

Execução das práticas em laboratório e sessões fotográficas.

\section{Textos de apoio}

A função dos textos de apoio é atender a dúvidas que surjam durante a utilização do material, bem como fornecer algumas informações adicionais, como fatos interessantes observados no cotidiano, que têm explicações arraigadas na Química e Bioquímica. A preocupação com a linguagem, buscando torná-la o mais compreensível possível, e com os exemplos fornecidos, tentando sempre aproximá-los do cotidiano dos estudantes e professores, foi constante durante o desenvolvimento do trabalho.

Os fundamentos teóricos foram divididos de acordo com as principais classes de biomoléculas estudadas em alimentos: lipídios, carboidratos, aminoácidos/proteínas e enzimas. A Figura 2 exemplifica a maneira de apresentação de cada uma dessas classes: 


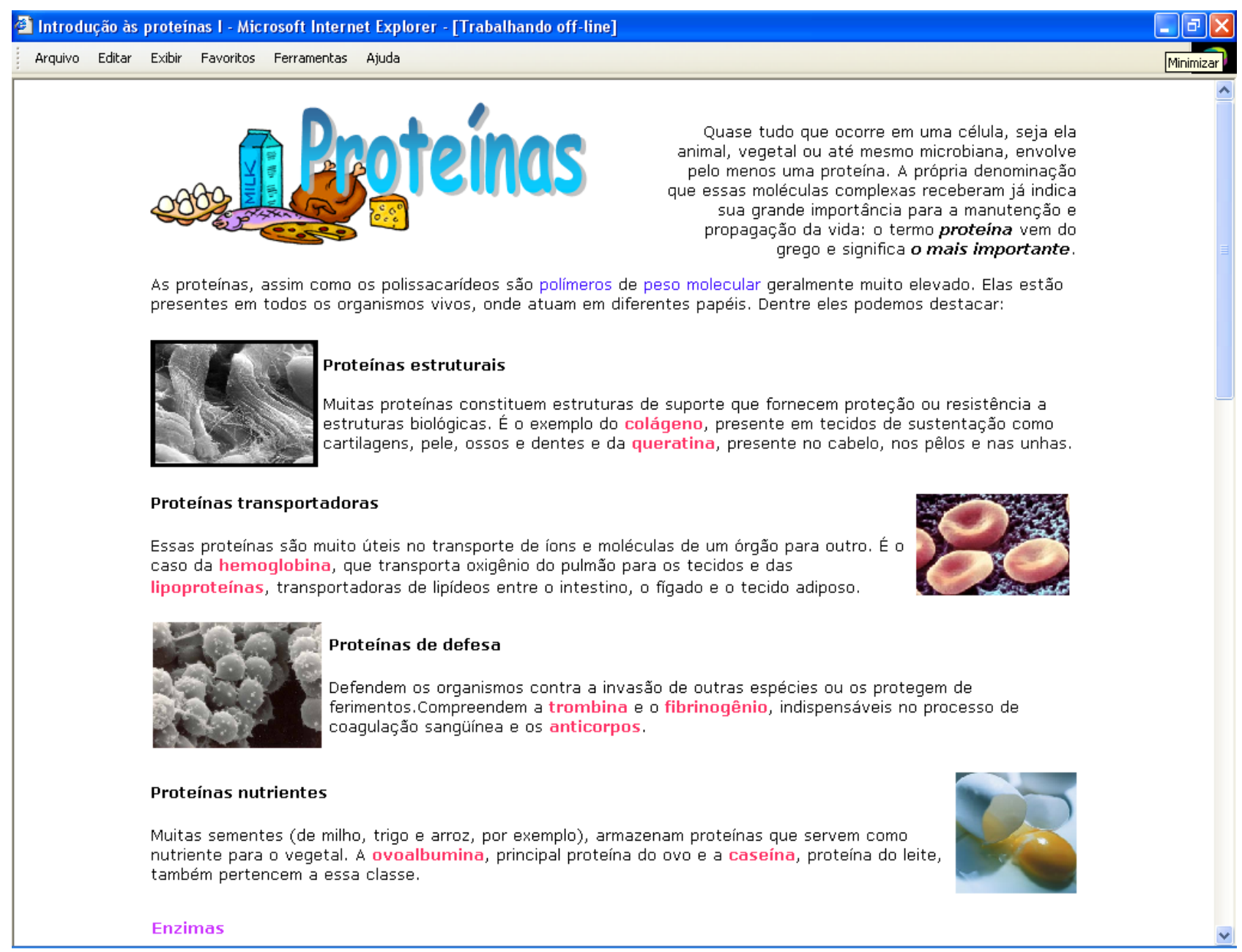

Figura 2. Página inicial do tópico "Aminoácidos e proteínas".

\section{Construção dos roteiros}

A proposta de construção de um curso prático de Bioquímica de Alimentos gerou a preocupação com a escolha de experimentos de execução relativamente simples e, quando possível, propondo a utilização de materiais e reagentes de baixo custo em substituição à vidraria e instrumental de laboratório, geralmente caros e de difícil aquisição. O grau de conhecimento necessário para o entendimento da prática também foi considerado, uma vez que o material em questão destina-se, inicialmente, a estudantes de nível médio.

Os roteiros também estão distribuídos de acordo com as classes de biomoléculas de interesse na área de alimentos, conforme descrito anteriormente. As atividades práticas podem ser acessadas separadamente, de acordo com 0 interesse $e$ necessidade do professor ou do estudante. Essa interatividade é muito importante, uma vez que permite que o texto oferecido adapte-se à necessidade particular de cada indivíduo ou de cada situação.

Ao todo foram elaborados 12 roteiros, que podem ser acessados separadamente através de links, conforme apresentado na Figura 3. 


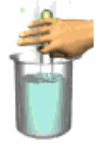

\section{Atividades práticas}

Esse trabalho traz uma série de atividades práticas, que têm o objetivo de ampliar seus conhecimentos e aguçar sua curiosidade acerca dos temas estudados. É fundamental, antes da realização de cada experimento, fazer uma leitura cuidadosa dos tópicos teóricos pertinentes à atividade a ser desenvolvida. Isso garantirá um melhor desempenho e, conseqüentemente, um melhor aprendizado. MAOS A OBRA!!!

\section{Lijpídios}

1. Determinaçã̃o de ácidos graxos livres e Índice de Acidez

2. $\underline{\text { Saponificaçãã }}$

3. Pesquisa de insaturaçỗes: adiç̧ã̃o de iodo

\section{Carioojdratos \\ 1.Caracterizaçẵo de carboidratos: teste de Molisch \\ 2. Diferenciação de aldoses e cetoses: reação de Seliwanoff \\ 3. Pesquisa de polissacarídeos: reaçẫo com o iodo \\ 4. Pesquisa de açúcares redutores: prova de Benedict}

\section{Proteingas}

1. Caracterização de aminoácidos e proteínas

2. Determinação do ponto isoelétrico da caseína

3. Propriedades gerais das proteínas

4. Extração e caracterizaçã̃o de enzimas

Figura 3. Apresentação das práticas disponíveis no material.

Em todos os roteiros foram adicionados links que permitem a consulta ao material teórico e às fotografias (Figura 4), etapas que serão discutidas ao longo desse trabalho.

Os roteiros das práticas encontram-se organizados de acordo com os seguintes itens: (1) Objetivos, (2) Princípios teóricos e (3) Procedimento Experimental. A Figura 4 ilustra os procedimentos experimentais da prática "Precipitação de proteínas". 


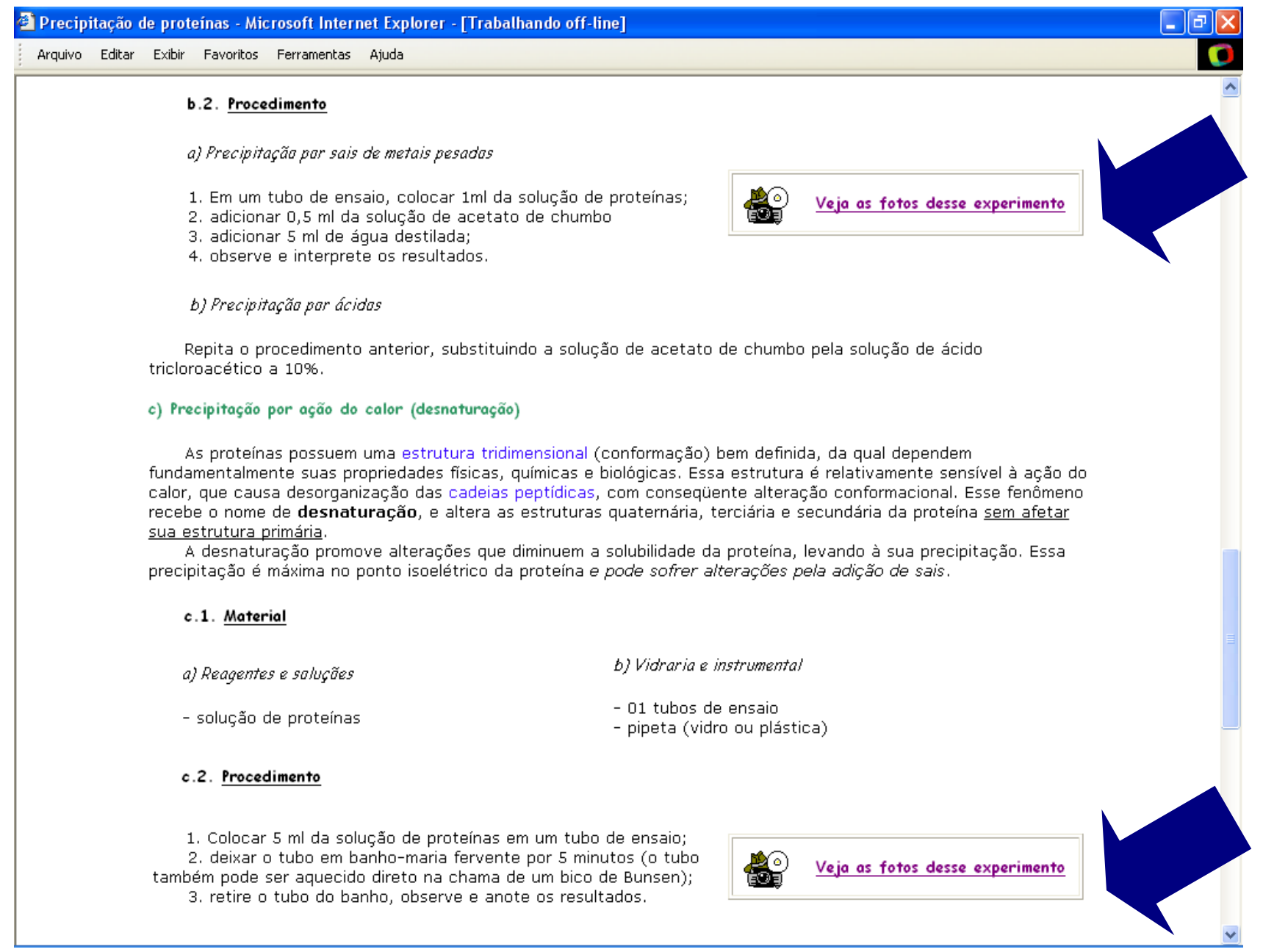

Figura 4. Procedimento experimental da prática "Precipitação de proteínas". Observe que as fotos podem ser acessadas através dos próprios roteiros.

\section{Experimentos fotografados}

Todos os experimentos propostos foram realizados em laboratório, de modo a verificar alguma possível incorreção nos roteiros, bem como propor modificações que melhorassem e facilitassem a execução da atividade proposta. Durante a realização das práticas, foram feitas fotografias. Todos os reagentes foram etiquetados, incluindo nome da substância, concentração e, quando necessário, periculosidade (Figura 5). 
Foto 1: Reagentes e equipamentos Foto 2: adicionar, aos dois tubos de ensaio, $1 \mathrm{~mL}$ da soluçắo de proteina

Foto 3: a um dos tubos, adicionar $0,5 \mathrm{~mL}$ de acetato de chumbo $20 \%$

Foto 4: ao outro tubo, adicionar $0,5 \mathrm{~mL}$ de ácido tricloroacético (TCA) $10 \%$

Foto 5: resultados

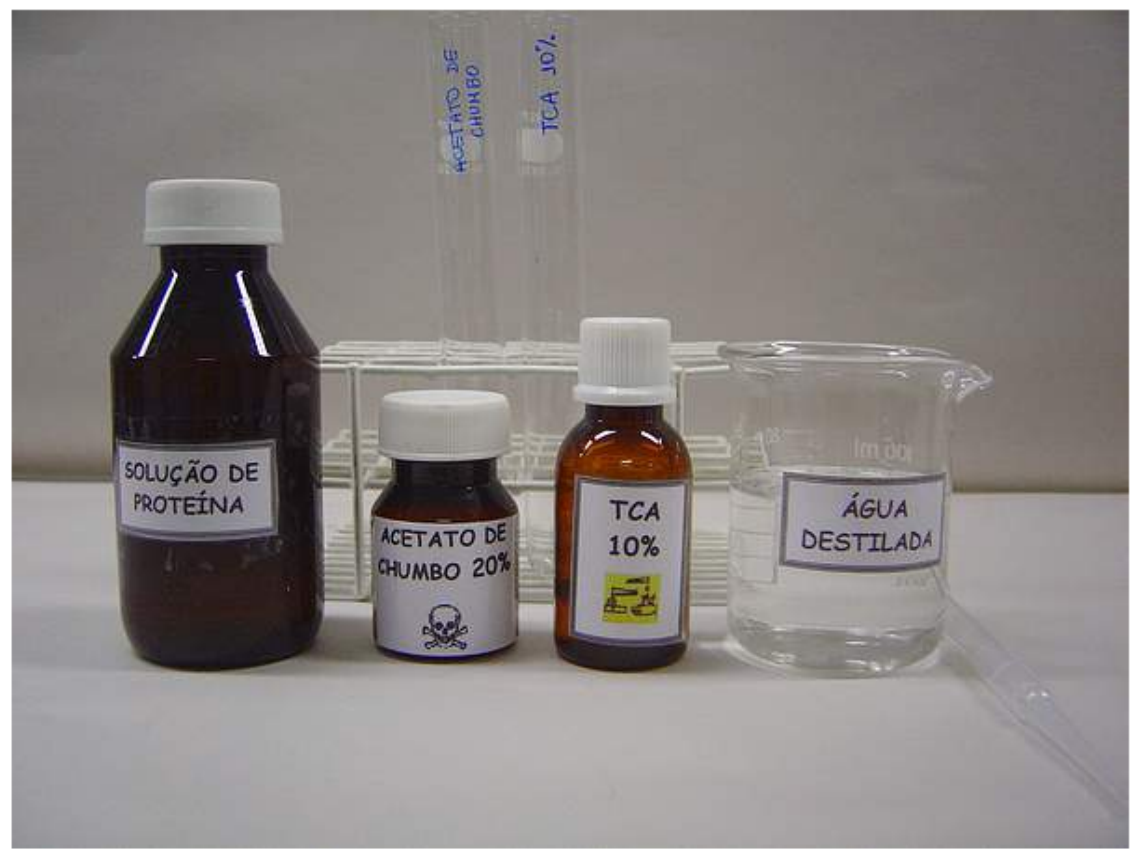

Figura 5. Prática "Propriedades gerais das proteínas". Observe os rótulos dos reagentes acetato de chumbo (tóxico) e TCA (corrosivo).

As fotos foram agrupadas em seqüências, que podem ser acessadas através de links encontrados ao final de cada roteiro (Figura 4). A própria visualização das fotos é interativa, uma vez que o usuário pode ir e voltar para as fotos de maior interesse, também através de links.

\section{CONSIDERAÇÕES FINAIS}

O trabalho foi idealizado e construído com base nas necessidades de professores e alunos de cursos relacionados à Ciência dos Alimentos. Porém, a contribuição da Bioquímica para a compreensão de diversas observações do cotidiano, aliada à curiosidade despertada pelos fenômenos relacionados aos alimentos, amplia significativamente as possibilidades de utilização desse material. Nesse sentido, o trabalho apresenta conteúdo adequado à proposta, possui facilidade de consulta e interatividade entre os temas abordados.

Cabe destacar, mais uma vez, a insuficiência na utilização da ferramenta aqui proposta de forma isolada da mediação do professor, uma vez que a teoria científica não deve ser resumida a um simples resultado de uma atividade experimental. Os estudantes devem ser introduzidos ao raciocínio científico e aos modelos da ciência, o que não pode ocorrer na ausência da atuação docente. De acordo com Driver e colaboradores [19]:

"Quem aprende precisa ter acesso não apenas às experiências físicas, mas também aos conceitos e modelos da ciência convencional. O desafio está em ajudar os aprendizes a se apropriarem desses modelos, a reconhecerem seus domínios de aplicabilidade, dentro desses domínios, a serem capazes de usá-los". (Driver et al, 1999, p. 34)

Em relação aos resultados da utilização desse material, atualmente está sendo desenvolvido um sistema de avaliação, que será disponibilizado no site com a intenção de promover melhorias, como correções e adição de novos conteúdos, 
facilidade essa proporcionada pela utilização de um meio rápido de comunicação como a internet.

\section{REFERÊNCIAS BIBLIOGRÁFICAS}

[1] M.V. Galliazi et al. (2001) Objetivos das atividades experimentais no ensino médio: a pesquisa coletiva como modo de formação de professores de ciências, Ciência \& Educação,7 (2), 249-263

[2] D. Hodson (1994) Hacia un enfoque más crítico del trabajo de laboratorio. Enseñanza de las Ciencias, 12, 299-313.

[3] O. Barberá ; P. Valdés (1996) El trabajo práctico en la enseñanza de las ciencias: una revisión. Enseñanza de las Ciencias,14, 365-379

[4] M. Giordan (2003) Experimentação por simulação. Textos LAPEQ (Faculdade de Educação, Universidade de São Paulo). Disponível em: http://pauling.fe.usp.br/artigos/exper simu/index.htm (acesso em 22/09/2004)

[5] BRASIL. MINISTÉRIO DA EDUCAÇÃO. Sistema de estatísticas educacionais (EDUDATABRASIL). Disponível em:http://www.edudatabrasil.inep.gov.br (acesso em $01 / 10 / 2006)$

[6] P. Saettler (1990) The evolution of American educational technology. Englewood, CO: Libraries Unlimited.

[7] M.D. Roblyer; J. Edwards; M. A. Havriluk (1997) Integrating educational technology into teaching. Nova Jersey: Prentice-Hall, p.1-79.

[8] V. F. Ferreira (1998) As tecnologias interativas no ensino. Química Nova, 21 (6), 780-786.

[9] R.G. Barreto (2003) Tecnologias na formação de professores: o discurso do MEC. Educação e Pesquisa, São Paulo, 29 (2), 271-286.

[10] (2002) O currículo: debates contemporâneos, Lopes / Macedo (Orgs.). São Paulo: Cortez, 216-236.

[11] BRASIL. MINISTÉRIO DA EDUCAÇÃO. Conversas com o professor sobre tecnologias educacionais. Disponível em: http://www.mec.gov.br/seb/pdf/conversas01.pdf (acesso em 10/10/2004)

[12] BRASIL. MINISTÉRIO DA EDUCAÇÃO (1998) Parâmetros Curriculares Nacionais, Terceiro e Quarto Ciclos do Ensino Fundamental. Brasília, MEC, 133-154.

[13] J. A. Valente (1993) Diferentes usos do computador na educação. Disponível em: http://upf.tche.br/ carolina/pos/valente.html (acesso em 11/12/2004).

[14] V.W. Setzer (1994) Computadores na educação: por quê, quando e como. Anais do $5^{\circ}$ Simpósio Brasileiro de Informática na Educação, Porto Alegre, 210-233.

[15] A. H. Bettega (2004) Educação continuada na era digital. 1 ed. São Paulo, Cortez. (Coleção Questões da nossa época, v.116)

[16] E. Galembeck et al. (2004) AMPc - Sinalização intracelular: um software educacional. Química Nova, 27 (3), 489-491.

[17] E. Galembeck; D.V. Macedo; B.B. Torres (2003) Different energy sources in sports Introductory software, Biochemistry and Molecular Biology Education, 31 (3), 204-208.

[18] L.H.A. Silva; Zanon, L.B. (2000) A experimentação no ensino de ciências. In:R.P.Schnetzler; R.M.R. Aragão.Ensino de ciências: fundamentos e abordagens. $1^{\text {a }}$ ed. Piracicaba: CAPES/UNIMEP, 2000, 120-153.

[19] DRIVER, R. et al. (1999) Construindo o conhecimento científico em sala de aula. Química Nova na Escola, 9, 31-40. 